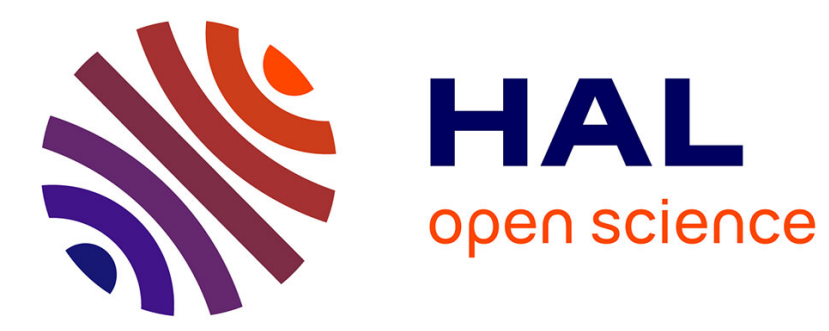

\title{
Strangeness measurements at the HADES experiment
}

Alexander Schmah

\section{To cite this version:}

Alexander Schmah. Strangeness measurements at the HADES experiment. Journal of Physics G: Nuclear and Particle Physics, 2010, 37 (9), pp.94004. 10.1088/0954-3899/37/9/094004 . hal-00600831

\section{HAL Id: hal-00600831 https://hal.science/hal-00600831}

Submitted on 16 Jun 2011

HAL is a multi-disciplinary open access archive for the deposit and dissemination of scientific research documents, whether they are published or not. The documents may come from teaching and research institutions in France or abroad, or from public or private research centers.
L'archive ouverte pluridisciplinaire HAL, est destinée au dépôt et à la diffusion de documents scientifiques de niveau recherche, publiés ou non, émanant des établissements d'enseignement et de recherche français ou étrangers, des laboratoires publics ou privés. 


\title{
Strangeness Measurements at the HADES Experiment
}

\author{
Alexander Schmah for the HADES collaboration \\ Technical University Munich, James-Franck Strasse 1, 85748 Garching, Germany \\ E-mail: a.schmah@gsi.de
}

\begin{abstract}
We report on HADES measurements of strange hadrons in the collision systems $\operatorname{Ar}(1.756 \mathrm{AGeV})+\mathrm{KCl}$ and $\mathrm{p}+\mathrm{p}$ at $3.5 \mathrm{GeV}$. Comparisons of $K_{s}^{0}$ transverse mass and rapidity spectra to IQMD transport model calculations give a strong hint to a repulsive kaon-nucleon potential. The effect of the potential shows up strongest at very low transverse momenta, which were measured by HADES with high statistics. Statistical model fits show a fair agreement to the particle yields measured in the heavyion data, apart from the $\Xi^{-}$yield which is off by more than an order of magnitude. Furthermore, first results from the $\Sigma(1385)^{+}$reconstruction in $\mathrm{p}+\mathrm{p}$ reactions are presented, showing an excellent agreement with published data.
\end{abstract}




\section{Introduction}

Heavy-ion collisions at SIS energies $(\mathrm{E}=1-2 \mathrm{AGeV})$ allow to create rather dense nuclear systems up to few times the saturation density, providing a favorable environment to study the hadron in-medium properties. The investigation of possible in-medium effects of hadrons, involving strange particles produced in heavy-ion collisions at SIS energies has been pursued during the last two decades and particular efforts have been devoted to the analysis of the $\mathrm{K}^{+}$and $\mathrm{K}^{-}$production.

The High Acceptance Di-Electron Spectrometer at the heavy-ion synchrotron SIS at GSI has been shown to be suited for precise measurements of strange hadrons [1]. A wide set of particles with single and double strange quark content, produced at or below the free NN-threshold energies, could be reconstructed in a small-sized heavy-ion reaction system and in $\mathrm{p}+\mathrm{p}$ collisions. For the first time, at SIS energies, the combined and inclusive identification of sub-threshold produced $K^{+}, K^{-}$and $\phi$ mesons in heavy-ion reactions was carried out [2]. As one of the results, the importance of the $\phi$ meson as a source of $K^{-}$was found to be much more relevant than expected. $K_{S}^{0}$ mesons have been measured as well and high statistics has been collected also in the low- $\mathrm{m}_{t}$ range for a broad rapidity interval. The detailed study of the $m_{t}$ distribution of $K_{S}^{0}$ down to very low transverse masses allows to discriminate more quantitatively among the theoretical calculations performed assuming different strength of the $K_{S}^{0}$-nucleus potential. As an example we show in the first section the comparison with the IQMD model.

The measured particle species allows to fit the particle yields by a statistical model. A clear deviation from the estimations by the model are found in case of the $\Xi^{-}$yield. The baryon chemical potential and the chemical freeze-out temperature was extracted and compared to other results in the $T-\mu_{B}$ plane.

In addition to the heavy-ion measurements, $\mathrm{p}+\mathrm{p}$ data at $3.5 \mathrm{GeV}$ have been measured and a large statistics has been collected. One of the goals of the elementary reaction program is the reconstruction of the $\Lambda(1405) / \Sigma(1385)$ in various decay channels. The understanding of the $\Lambda(1405)$ is also a starting point for the search of deeply bound kaonic states [3]. As a first step and result of this analysis the reconstruction of the $\Sigma(1385)^{+}$will be presented in the last section.

\section{In medium modification of $K^{0}$ mesons in $\mathrm{Ar}+\mathrm{KCl}$ reactions}

The properties of neutral kaons $\left(\mathrm{K}_{S}^{0}\right)$, produced in heavy-ion collisions, can be studied in order to exctract a kaon-nucleon potential. The in-medium potential is expected to be repulsive for both $\mathrm{K}^{+}$and $\mathrm{K}^{0}$. As far as the $\mathrm{K}^{0}$ in-medium properties are concerned, recent results extracted from pion-induced reactions should be mentioned [4]. High statistics and a large phase space coverage of $\mathrm{K}_{S}^{0}$, produced in $\mathrm{Ar}+\mathrm{KCl}$ reactions at $1.756 \mathrm{AGeV}$, allows a differential comparison of the HADES data to transport model calculations with and without a kaon-nucleon potential. 


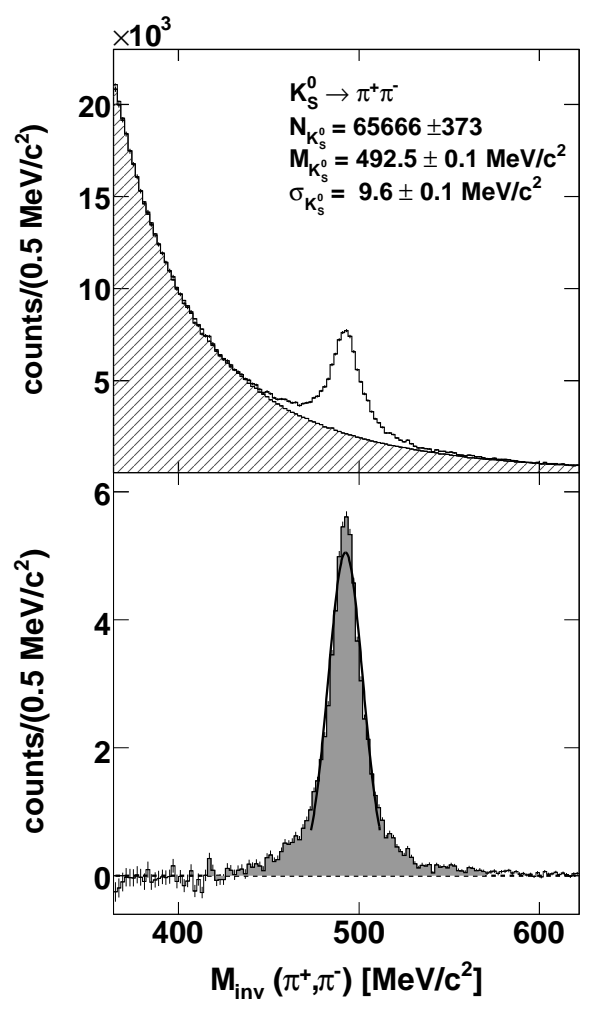

Figure 1. Invariant-mass distribution of $\pi^{+}-\pi^{-}$pairs (top). The combinatorial background (shaded area) is obtained by the mixed-event technique. The backgroundsubtracted distribution (bottom) shows clearly he $\mathrm{K}_{S}^{0}$ meson signal (grey area with a Gaussian fit).

About $66.000 \mathrm{~K}_{S}^{0}$ mesons were reconstructed in the decay channel $K_{S}^{0} \rightarrow \pi^{+}+\pi^{-}$ with a signal to background ratio varying from 0.3 to 2.0 depending on the transverse momentum and rapidity. Figure 1 shows the integral invariant mass distribution of $\pi^{ \pm_{-}}$ pairs after cuts on the production and decay vertices were applied in order to suppress background events. The remaining combinatorial background was subtracted by using the mixed event techniques. The mixed event background is shown in Fig. 1 as a grey shaded area. For the efficiency and acceptance corrections full scale simulations were performed by using the GEANT3 package [5].

Fig. 2 (left) shows the transverse mass $m_{t}-m_{K_{s}^{0}}$ spectra of $\mathrm{K}_{s}^{0}$ by empty symbols after background subtraction and correction for acceptance and efficiency for different $y_{c . m}$. bins. A fit according to

$$
\frac{1}{m_{t}^{2}} \frac{d^{2} N}{d m_{t} d y}=C(y) \exp \left(-\frac{\left(m_{t}-m_{0}\right) c^{2}}{T(y)}\right)
$$

has been applied to the data. Using this Boltzmann parameterization an inverse slope parameter $T_{B}(y)$ can be determined as a function of the rapidity. Fitting the $T_{B}(y)$ distribution with the function $T_{B}(y)=\frac{T_{\text {eff }}}{\cosh (y)}$ the parameter $T_{\text {eff }}$ can be determined. This represents the inverse slope at mid-rapidity and corresponds to an effective temperature at the kinetic freeze-out stage as was found to be $92 \pm 2 \mathrm{MeV}$. The rapidity densitiy distribution was calculated by integrating the fitted Boltzmann distributions from 0 to 

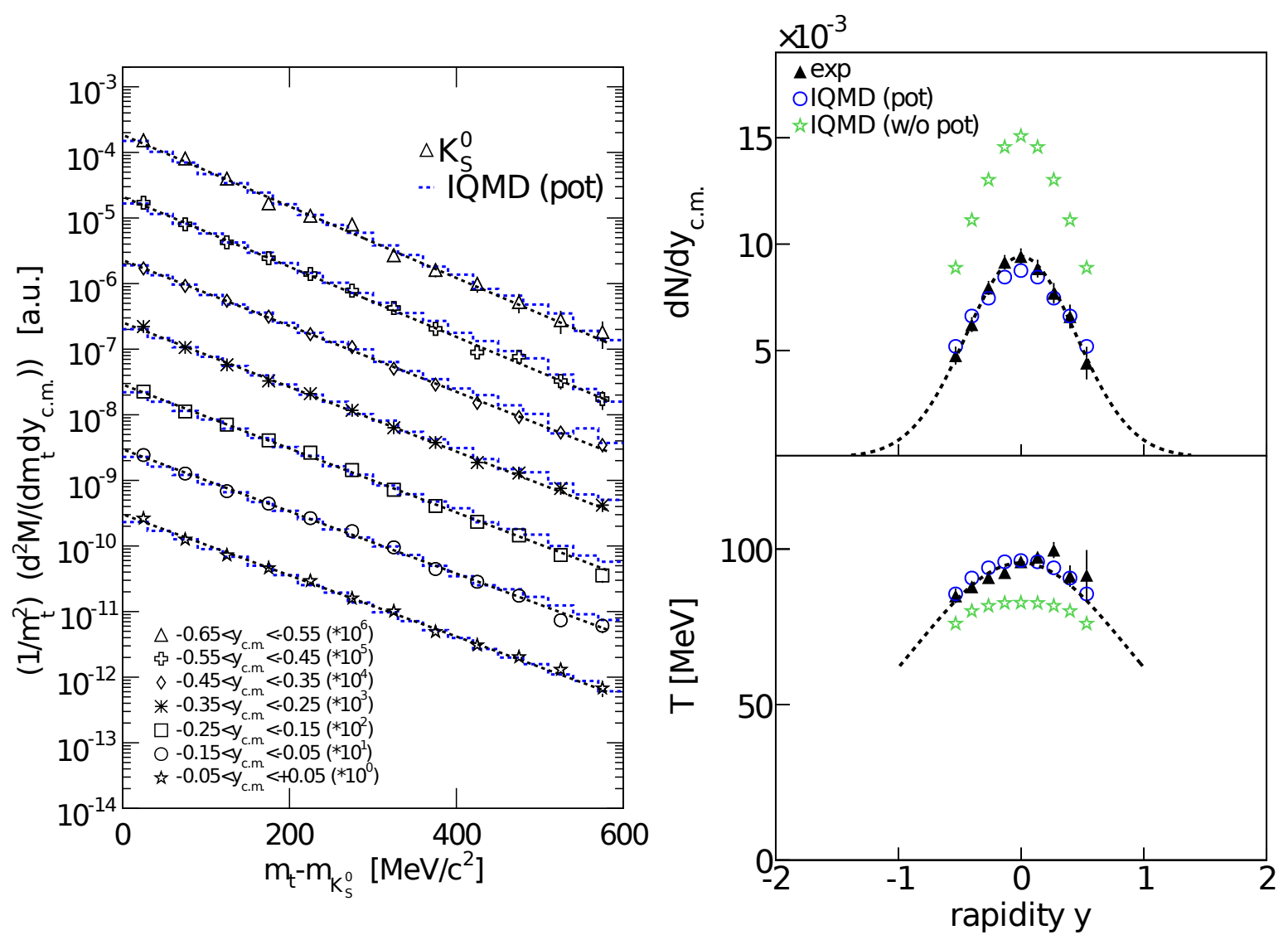

Figure 2. Left: Transverse mass spectra of $K_{s}^{0}$ mesons from $\mathrm{Ar}+\mathrm{KCl}$ reactions at 1.756 AGeV for different rapidity bins. The overlayed blue histogram shows a IQMD transport model calculation with a kaon-nucleon potential strength of about $40 \mathrm{MeV}$. The dashed curves are fits by Eq. 1. Right: Rapidity distribution (top) and inverse slope parameters (bottom) as a function of the center-of-momentum rapidity. IQMD transport model calculations without kaon-nucleon potential (green stars) and with potential (open blue circles) are overlayed.

$\infty$. The transverse mass spectra in Fig. 2 as well as the $\mathrm{dN} / \mathrm{dy}$ and $T_{B}$ distributions, shown in Fig. 2 (right), are compared to IQMD transport model calculations. Among other models, the IQMD model was used in the past to compare with KaoS and FOPI data [6]. The IQMD simulations have been calculated assuming a selection of the impact parameter $\mathrm{b}<6 \mathrm{fm}$. This value was fixed by a normalization to the pion yield. Both, the $\mathrm{dN} / \mathrm{dy}$ and the inverse slope distributions, are better reproduced by a IQMD calculation with a $K_{s}^{0}$-nucleus potential of about $40 \mathrm{MeV}$. Within the errors the $\mathrm{K}^{+}$ meson transverse mass spectra agree with the $K_{s}^{0}$ distributions [7].

A more detailed comparison of $K_{s}^{0} p_{t}$-spectra to the IQMD model calculations to extract the $\mathrm{KN}$ potential strength is shown in [7]. 


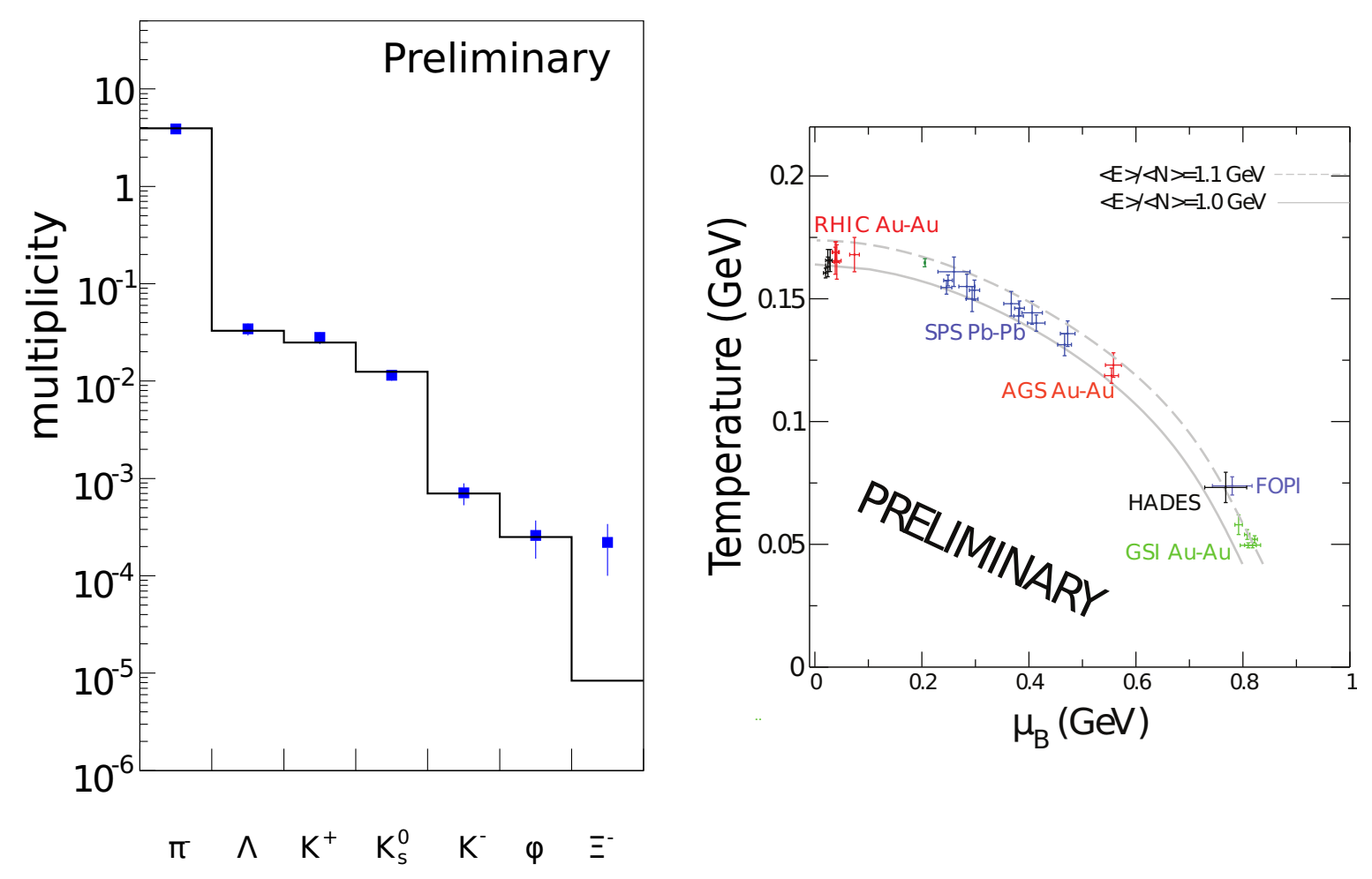

Figure 3. Left: Particle multiplicities in $\mathrm{Ar}+\mathrm{KCl}$ reactions at $1.756 \mathrm{AGeV}$ together with fit by the THERMUS statistical model to the data. Right: Chemical freeze-out temperature as a function of the baryon chemical potential $\mu_{B}$. The shown data ranges from RHIC down to SIS energies. The included HADES data point corresponds to the result of the statistical model fit shown left picture.

\section{Statistical model fit and chemical freeze-out}

In the reaction system $\mathrm{Ar}+\mathrm{KCl}$ at $1.756 \mathrm{AGeV}$ several particles species were measured within a large kinematic range, allowing to extract their multiplicities with small extrapolation uncertainties. In the past it was shown that the statistical model is able to describe relative particle yields from heavy-ion collisions fairly good [10]. Possible fit parameters of the statistical model are the chemical freeze-out temperature $T$, the baryon-chemical potential $\mu_{B}$, the volume of the fireball $\mathrm{R}$ and the strangeness correlation radius $R_{c}$. In Fig. 3 (left) a fit of the THERMUS [9] model to the HADES $\mathrm{Ar}+\mathrm{KCl}$ data is shown. The $\mathrm{K}^{ \pm}, \mathrm{K}_{S}^{0}, \pi^{-}$and $\Lambda$ hadrons were measured in a large acceptance, whereas the $\phi$ and $\Xi^{-}$were measured only in a small kinematical range. Except for the $\Xi^{-}$data point all other particle yields were fitted within the error bars by the statistical model. The $\Xi^{-}$analysis and the comparison to the statistical model is described in detail elsewhere [11].

We extracted the following values for the chemical freeze-out conditions: $T \approx 73$ $\mathrm{MeV}, \mu_{B} \approx 770 \mathrm{MeV}, R_{c} \approx 2.4 \mathrm{fm}$ and $R \approx 4.9 \mathrm{fm}$. The $T$ and $\mu_{B}$ value is plotted in 
Fig. 3 (right) together with other data from SIS, AGS, SPS and RHIC experiments [12]. The HADES data is consistent with results from the FOPI collaboration at a similar beam energy. The line with a constant mean energy per nucleon with a value of about $\langle E>/\langle N>\approx 1.1 \mathrm{GeV}$ is in agreement with all SIS data points.

\section{Production of $\Sigma(1385)$ and $\Lambda(1405)$ in $\mathbf{p}+\mathbf{p}$ reactions at $3.5 \mathrm{GeV}$}

In April/May 2007 about 1.2 billion $\mathrm{p}+\mathrm{p}$ reactions were collected with HADES. As a first level trigger, three particles in the META detector were required. The experimental setup was nearly identical to the one of the $\mathrm{Ar}+\mathrm{KCl}$ beam time. As an additional detector, a time-of-flight wall was installed $7 \mathrm{~m}$ downstream of the target, which covers polar angles from 0.3 to 7 degrees. Since a start detector was not installed, the event start time was recalculated, using the momentum information of at least one identified particle. For this physics analysis the pions and protons were identified via the energy loss information of the multi-wire drift chambers, whereas for the kaons additional information as the energy loss in the time-of-flight detectors and the calculated particle mass were used. One of the analysis goals is the reconstruction of the $\Lambda(1405)$ in all its decay channels:

$$
\begin{aligned}
& \Lambda(1405) \rightarrow \Sigma^{+}+\pi^{-} \rightarrow n+\pi^{+}+\pi^{-}, \\
& \Lambda(1405) \rightarrow \Sigma^{-}+\pi^{+} \rightarrow n+\pi^{-}+\pi^{+} \\
& \Lambda(1405) \rightarrow \Sigma^{0}+\pi^{0} \rightarrow \Lambda+\gamma+\pi^{0} .
\end{aligned}
$$

The particle is well known since many decades but its internal structure is still unknown. Calculations and recent experimental findings show different lineshapes of the $\Lambda(1405)$ depending on the production and decay channels.

The $\Lambda(1405)$ signal is overlapped in all charged $\Sigma \pi$ decay channels by the $\Sigma(1385)^{0}$ resonance, which has identical daughter particles. Even in the $\Sigma^{0} \pi^{0}$ decay channel it is not possible with the actual HADES setup to fully distinguish between the two particles as it was done for example in [13]. To get rid of background an exclusive analysis of the production channel $p+p \rightarrow \Lambda(1405) / \Sigma(1385)^{0}+K^{+}+p$ is inevitable. This means a measurement of all charged particles and a reconstruction of intermediate and missing neutral particles using the missing mass and invariant mass technique.

Since the production and decay chain of the $\Sigma(1385)^{+}$is comparable to the ones of the $\Lambda(1405)$, its reconstruction can be used as a benchmark for the $\Lambda(1405)$ analysis. Furthermore the lineshape of the $\Sigma(1385)^{+}$and its cross section is important for the later subtraction of the $\Sigma(1385)^{0}$ signal from the $\Lambda(1405)$ spectrum. The following production and decay channel was investigated:

$$
p+p \rightarrow \Sigma(1385)^{+}+K^{+}+n \rightarrow\left(\Lambda+\pi^{+}\right)+K^{+}+n .
$$

In Fig. 4 the reconstructed missing neutron and the $\Lambda$ hyperon are shown. Figure 5 shows the invariant mass of $\Lambda+\pi^{+}$after the selection of the missing neutron and the $\Lambda$ and the subtraction of a simulated phase space background. The reconstructed 

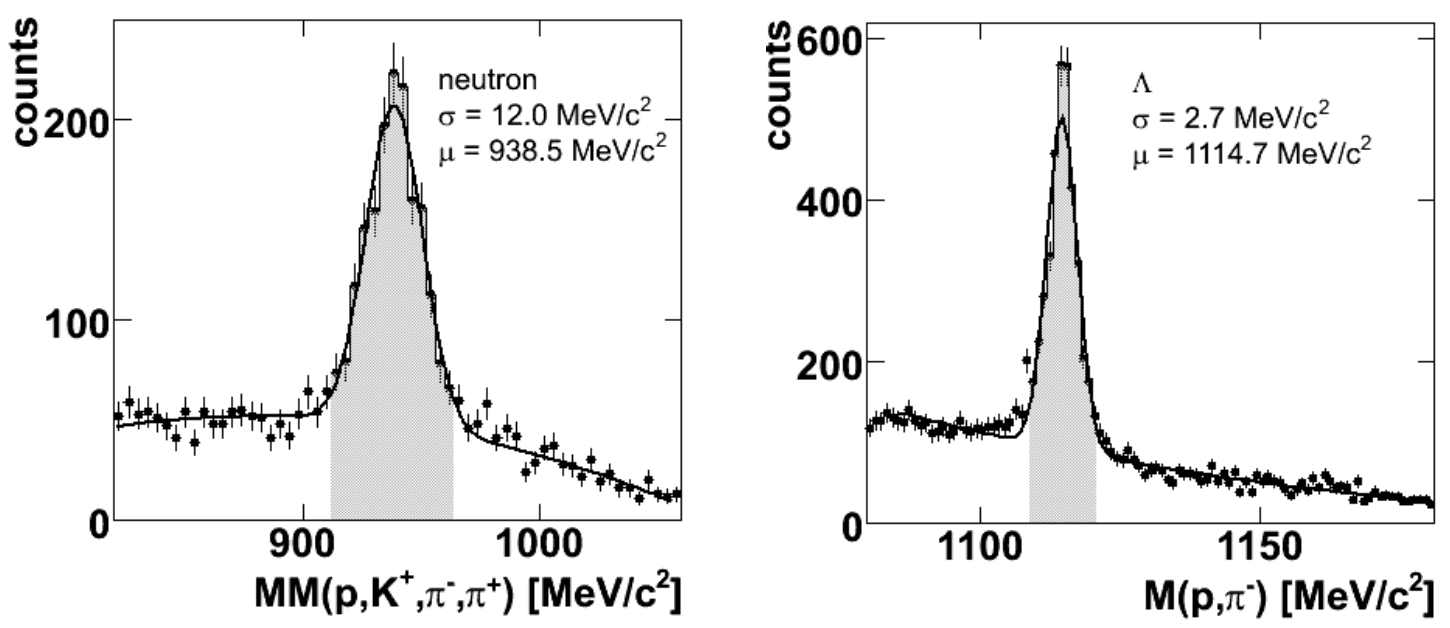

Figure 4. Left: The missing neutron in the decay is calculated by the missing mass of all charged particles. Right: The invariant mass of proton and $\pi^{-}$shows the $\Lambda$ signal. The grey shaded area shows the cut regions on the two particles for the $\Sigma(1385)^{+}$ reconstruction.

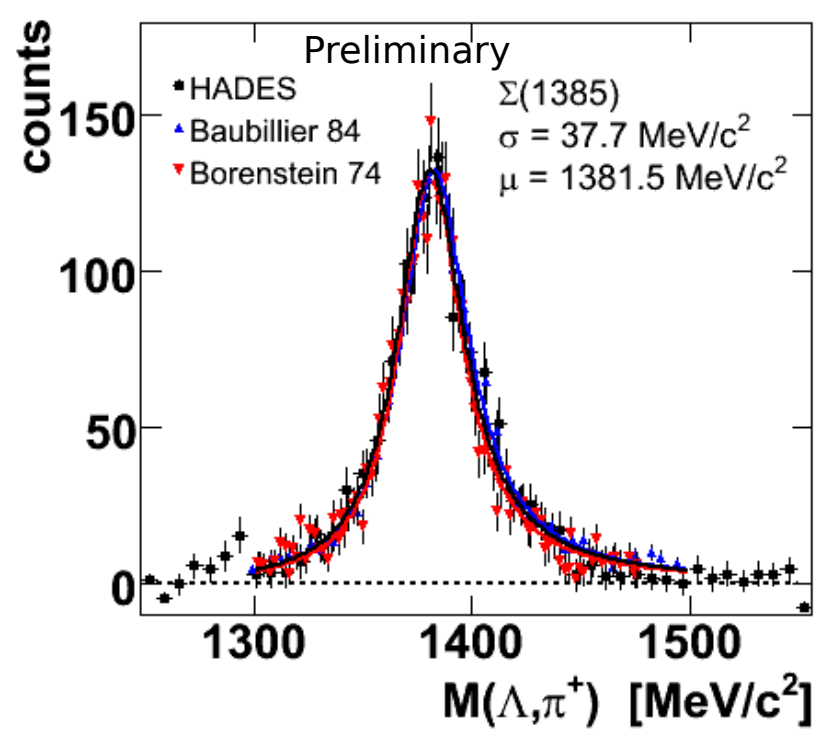

Figure 5. Invariant mass of $\Lambda$ and $\pi^{+}$after a selection of a missing neutron (see text). The non-resonant background is already subtracted. The black rectangles shows the HADES data from $\mathrm{p}+\mathrm{p}$ reactions in comparison to the normalized data from [14] and [15]. The width and pole mass position corresponds to the Breit-Wigner fit shown in the figure. 
$\Sigma(1385)^{+}$shows a nice agreement to the lineshapes of previously published data $[14,15]$. The $\Lambda(1405)$ analysis was performed in a similar way. First results show the feasibility to reconstruct the resonance in all $\Sigma \pi$ decay channels.

\section{Summary and outlook}

We presented high resolution $K_{s}^{0}$ data from $\mathrm{Ar}+\mathrm{KCl}$ reactions at $1.756 \mathrm{AGeV}$ in comparison to IQMD transport model calculation. The simulation with a kaon-nucleon potential of about $40 \mathrm{MeV}$ show a good agreement with the data. Statistical model calculations were performed and fitted to the yields of the measured hadrons in the $\mathrm{Ar}+\mathrm{KCl}$ beam time. The extracted $\mathrm{T}$ and $\mu_{B}$ values are in good agreement with the world data. In $\mathrm{p}+\mathrm{p}$ reactions at $3.5 \mathrm{GeV}$, the reconstruction of the $\Sigma(1385)^{+}$ was presented and compared to reference data. The result shows the feasibility for an $\Lambda(1405)$ analysis with HADES.

The upgrade of the HADES experiment with the high resolution RPC detectors, an up to ten times faster DAQ system, and improved multi-wire-drift chambers will be completed within this year. An already installed forward wall hodoscope will deliver important information for the event plane reconstruction and the reaction centrality. With this setup it is planned to take data using medium and heavy sized reaction systems from 2010 on. Furthermore, experiments with pion beams are scheduled to study strangeness production in cold matter.

The collaboration gratefully acknowledges the support by BMBF grants 06DR135, 06FY171, 06MT238 T5, and 06MT9156 TP5 (Germany), by the DFG EClust 153, by GSI TMKRUE, by grants MSMT LC07050 and GA ASCR IAA100480803 (Czech Rep.), by grants RII3-CT-2005-515876, FPA2006-09154, and CPAN:CSD2007-00042 (Spain).

\section{References}

[1] A. Schmah, doctoral thesis, Darmstadt (2008).

[2] G. Agakishiev et al. [HADES Collaboration], Phys. Rev. C 80 (2009).

[3] T. Yamazaki, Y. Akaishi, Phys.bLett. B535, 70 (2002).

[4] M.L. Benabderrahmane et al. (FOPI), Phys. Rev. Lett. 102, (2009).

[5] GEANT 3.21, http://consult.cern.ch/writeup/geant/ (1993).

[6] C. Fuchs, Prog. Part. Nucl. Phys. 56 (2006).

[7] G. Agakishiev et al. [HADES Collaboration], In-Medium Modification of $K^{0}$ in Relativistic Heavy Ion Collisions, in preparation

[8] G. Agakishiev et al., Overview of strangeness production in $\mathrm{Ar}+\mathrm{KCl}$ reactions at $1.756 \mathrm{AGeV}$, in preparation

[9] S. Wheaton et al., Comput. Phys. Commun. 180 (2009).

[10] A. Andronic, P. Braun-Munzinger and J. Stachel, Nucl. Phys. A 772, 167 (2006).

[11] G. Agakishiev et al. [HADES collaboration], Phys. Rev. Lett. 103 (2009).

[12] J. Cleymans, priv. communication (2009)

[13] I. Zychor et al., Phys. Lett. B660 (2008), 167

[14] M. Baubillier et al., Z. Phys. C 23 (1984) 213.

[15] S. R. Borenstein et al., Phys. Rev. D 9 (1974) 3006. 